\title{
ISO and SOHO - First Results
}

M.C.E. Huber reports on the EPS-10 symposium Results from the ESA Satellites ISO and SOHO which reviewed spectacular first results that cover the universe from comets to the cosmos, including an integral investigation of the Sun.

"When you look at a star, you sometimes don't realise that the Sun is a star as well, and that the star is actually part of the Milky Way." This remark, made by Marc Sauvage (Saclay) during his talk on ISO and infrared emission from normal galaxies captured perfectly the multidisciplinary spirit of the symposium Results from the ESA Satellites ISO and $\mathrm{SOHO}$ that was held on the last day of the EPS-10 General Conference. The symposium offered a condensed overview of observations and research carried out with the two most recent scientific European Space Agency (ESA) satellites: the Infrared Space Observatory, ISO, and the Solar and Heliospheric Observatory, SOHO, which were launched last year within two weeks of each other - a rarity for space science.

A short introduction stressed the decisive role played by the wide scientific community in Europe in defining the con-

M.C.E. Huber heads ESA's Space Science Department and chairs the Joint EPS - European Astronomical Society Astrophysics Division. tent of ESA's scientific programme. The advent of space missions addressing fundamental physics, notably the dogmas of general relativity, was also mentioned.

The ISO and SOHO facilities, including access by the community to these two observatories, were briefly described by the respective ESA Project Scientists. Operation of the $5 \mathrm{~m}$ high, 2.5 tonne ISO infrared telescope has been essentially trouble-free since its launch in November 1995 (M.F. Kessler, Villafranca). All four instruments (two cameras and two spectrometers) are working well, the only problem being some reduction in sensitivity owing to cosmic-ray impacts. Operation will be extended from 18 to 24 months since the bleed-off rate of the telescope's 2000 litres of superfluid helium cryogen is lower than expected. The $3.7 \mathrm{~m}$ high, 1.9 tonne SOHO satellite carries out roundthe-clock observations of the Sun (including 12 hours in real time) from the Lagrangian point. It has been fully commissioned for six months and all instruments are performing well (V. Domingo, Greenbelt).

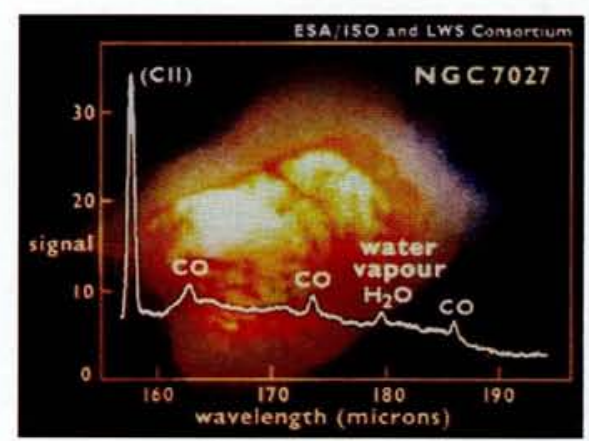

Fig. 1. ISO Long-Wavelength Spectrometer spectrum of the planetary nebulae NGC 7027 superimposed on a Hubble Space telescope image. The spectrum reveals the presence of water vapour in this carbon-rich cloud of dust and gas.

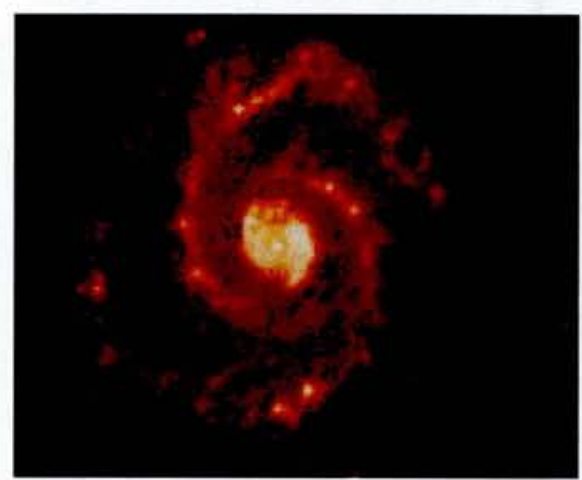

Fig. 2. Star formation. An ISO infrared image of the M51 "Whirlpool" galaxy taken by ISOCAM at a wavelength of $15 \mu \mathrm{m}$. Bright spots in the spiral arms correspond with warm dust clouds where star formation is proceeding on a large scale.

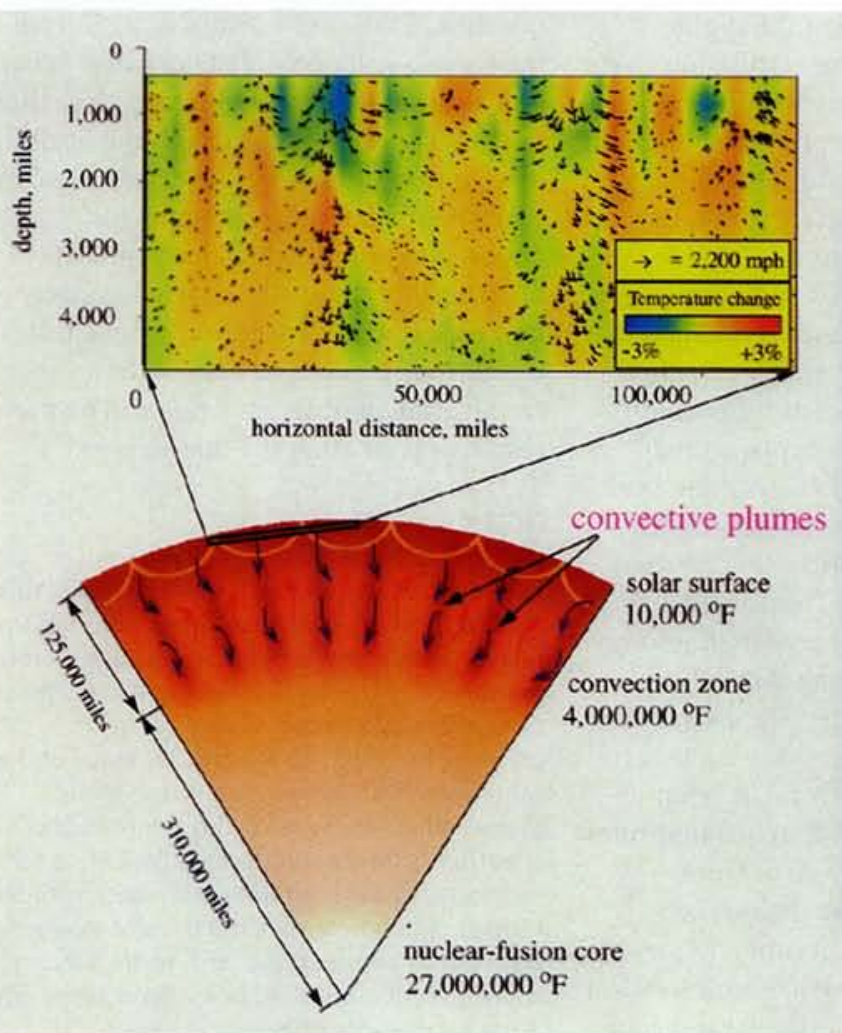

Fig. 3. (below). Rho Ophiuchi, a nearby interstellar dust cloud. It is completely opaque to visible light, where one only sees the few stars between Earth and the cloud. Thanks to the more penetrating power of infrared light, ISO's ISOCAM reveals details of the cloud's interior. Colour composite: blue for $7 \mu \mathrm{m}$ radiation; red for $15 \mu \mathrm{m}$ radiation. The stars are recently formed young stars.

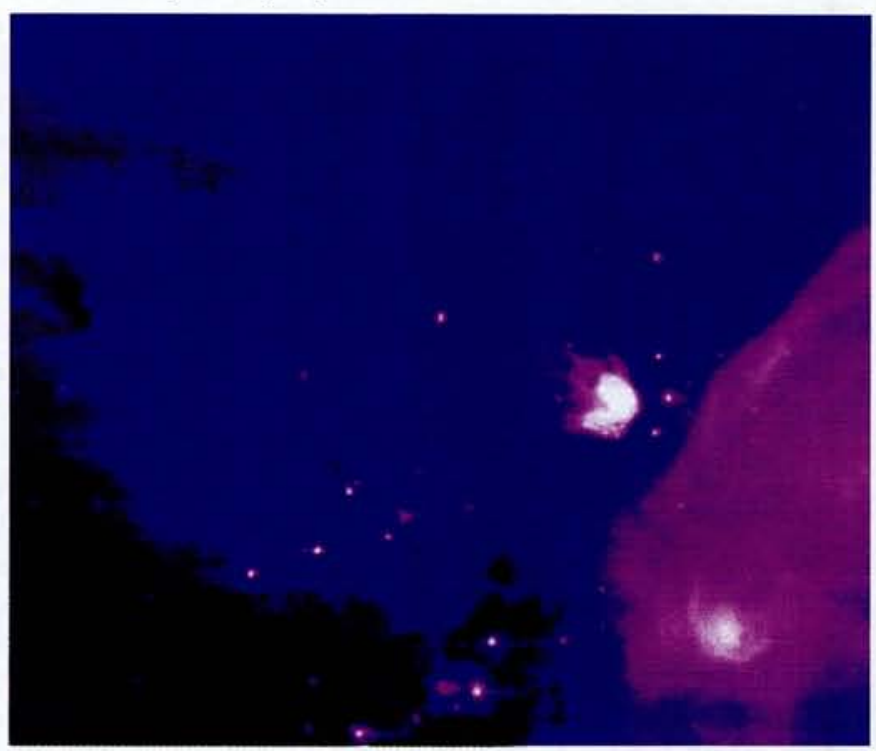

Fig. 4. (left). Convective flows beneath the outer layers of the Sun's surface as determined by $\mathrm{SOHO}^{\prime} \mathrm{S} \mathrm{MDI}$ instrument. 


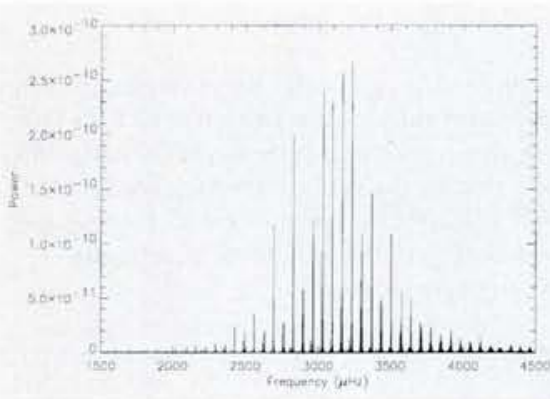

Fig. 5. Fourier-analysis of solar pressure oscillations ( $p$ modes), as observed by SOHO's VIRGO experiment, showing the spectrum in the region of the five-minute period. These data were obtained by an instrument observing the entire solar hemisphere facing Earth, i.e., by observing the Sun in the same way as a star.

First findings were then presented in a series of invited talks, equally shared between the two missions. Those on ISO covered stellar evolution and starformation in the Milky Way, the infrared emission from normal galaxies and ISO's observations of active and ultraluminous galaxies. SOHO reports covered the physical processes in the solar atmosphere, as seen through ultraviolet observations, new insights into corona and heliosphere through ultrasensitive observations in the optical domain, the diagnostic power of mass spectroscopy of the solar wind, and the structure and dynamics of the solar interior as revealed by helioseismology.

The figures illustrate highlights from the first results. One of the early surprises from ISO was the detection of an emission line from water vapour in the young planetary nebula, NGC 7027 (Fig. 1). This was unexpected as the object is carbon-rich and the oxygen was thought to be tied up in carbon dioxide rather than in water. Since then, ISO has detected many different emission lines from water vapour across most of its wavelength range in a variety of sources, including Herbig Haro objects and the variable star W Hydrae.

ISO's increased spatial resolution as compared to the earlier infrared sky survey made by the IRAS satellite is being demonstrated by a variety of spectacular maps. Examples include: maps (Fig. 2) at all ISO wavelengths of the resolved spiral galaxy M51, giving detailed information about the star formation underway in the spiral arms; and maps (Fig. 3) of the rho Ophiuchi dark cloud (which is completely obscured in the visible spectral region) at wavelengths of 7 and 15 microns showing a large number of young stars and regions so dense that they are opaque even to ISO.

$\mathrm{SOHO}$ has made measurements of temperature and flow velocity in the solar corona. The coronagraphs on SOHO have provided the first measurements in the region where the solar wind is accelerated. (The solar wind is the hot plasma that is released by the Sun - sometimes in steady streams, sometimes in gusts - and reaches the Earth after two or three days when it affects the geomagnetic environment.) The measurements show that the wind is accelerated from a few $\mathrm{km} / \mathrm{s}$ near the solar surface to hundreds of $\mathrm{km} / \mathrm{s}$ at $2.0-2.5$ solar radii. $\mathrm{SOHO}$ is also providing the first detailed view of the outer layers of the solar interior (Fig. 4). Data from the Michelson Doppler Imager instrument show that the uppermost convection cells (the so-called super-granule cells) are found to be flat and pancake-shaped. In the generally accepted mixing-length theory it was previously assumed that the cells were deep and shaped like spheres.

The participants were anxious to learn whether SOHO's helioseismology experiments had already yielded some hints about the structure of the innermost core of the Sun that could shed light on the neutrino problem. Existing helioseismology data, contrary to neutrino data, is well explained by the Standard Solar Model. Teo Roca-Cortés (Tenerife) reported that gravity modes (which carry information on the solar core) have not yet been seen. He expressed optimism though, since the

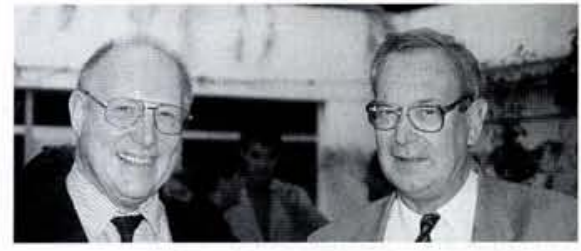

M.C.E. Huber, on the left, with G. Tammann from Basel who gave the plenary talk Expansion of the Universe.

noise level found in the SOHO oscillation data is more than 10-times lower than in those obtained from the ground.

An example of the oscillations spectrum around the five-minute period is given in Fig. 5. The spectrum shows eigenfrequencies of pressure modes which predominantly probe the outer layers of the Sun. While the "lines" at the lowfrequency end are extremely sharp, a significant broadening is noted at higher frequencies: apparently the lifetime of the high-frequency modes is shorter than that of low-frequency modes (3-4 days versus 34 months) and, accordingly, the frequency is more uncertain at high frequencies. Long observing periods will be needed to accurately determine the oscillation frequencies and line profiles, and to follow their change with the Sun's magnetic cycle. There also appears to be a hyperfine structure on high-frequency peaks that may be due to rotation below the convection zone.

\section{Unique Opportunities with Storage Rings}

R. Schuch and M. Larsson from Stockholm University report that the EPS-10 symposium Physics with Ion

Storage Cooler Rings demonstrated the unique opportunities offered by ion storage cooler rings.

With its high currents of cooled ions and cold electrons, the storage ring provides unique conditions for precision investigations in atomic, molecular, and nuclear physics. In reviewing the various cooling methods for stored ions with $\mathrm{MeV}$ kinetic energy (stochastic, electron and laser cooling), the EPS-10 symposium Physics with Ion Storage Cooler Rings emphasized recent developments, notably in electron cooling where meV electron temperatures are reached by adiabatically expanding the electron beam, first implemented at Stockholm's CRYRING.

Andreas Wolf (Heidelberg) described how electron coolers in storage rings serve two purposes: they act as cooling agents for the stored ion beam and as targets in electron-ion collisions. The electron and ion beams move with the same average velocity when the ion beam is phase-space cooled. If the electron-beam velocity is changed so that the electrons move faster (or slower) than the ions, electron-ion collisions at a well-defined energy down to the meV range are induced.

Collisions between electrons and atomic ions lead to recombination if the free electron can deposit its energy to the ion while being captured, and if the capture process can be stabilized. For a bare ion containing no electrons, capture and stabilization occurs in one step by the

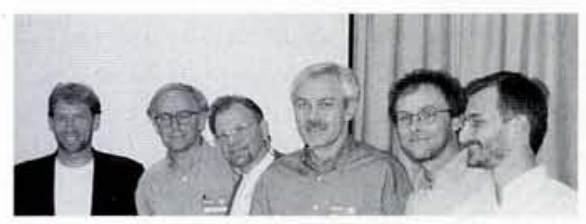

The speakers and organizers of the EPS-10 symposium Physics with lon Storage Cooler Rings From left to right: J. Hangst (Aarhus), M. Larsson (Stockholm), J. Eades (CERN), R. Schuch (Stockholm), T. Stöhlker (Darmstadt), and A. Wolf(Hedielberg). 